\title{
Análisis de prevalencia de la mortalidad atribuible a causas conocidas de muerte súbita en Chile, población de 1 a 35 años, 2000-2010.
}

\author{
Benjamín Donoso ${ }^{1}$, Francisco Bengoa ${ }^{2}$, Francisco López $^{2}$, Daily Piedra ${ }^{3}$, Cristián Clavería ${ }^{4}$
}

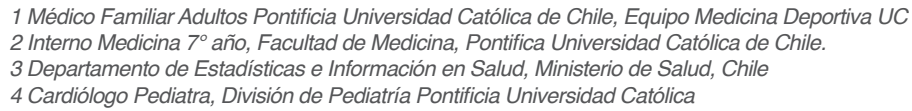

Antecedentes: Pocas situaciones son más devastadoras que los casos de pacientes, sin patología previa diagnosticada, que presentan muerte súbita (MS), especialmente durante la práctica deportiva. La mayor parte de las MS son consecuencia de una patología cardíaca subyacente no diagnosticada, definida como muerte súbita cardíaca (MSC).

En Chile no existen reportes que analicen la epidemiología de la MSC. La literatura Internacional describe la relación entre patologías cardíacas bien definidas y ejercicio competitivo como gatillante de la MSC.

Objetivo: Caracterizar la prevalencia de patologías cardíacas y sus subtipos reconocidos como causas de MSC en el grupo etario de 1 año a 35 años.

Métodos: Se analizaron los registros del Departamento de Estadísticas e Información del Ministerio de Salud y los certificados de defunción de todos los fallecidos entre los años 2000-2010 del grupo etario de 1 año a 35 años. Se seleccionaron datos de los fallecidos con diagnósticos relacionados con MSC de acuerdo al Código Internacional de Enfermedades (CIE-10).

Resultados: de un total de 57.979 fallecidos, 1131 $(1,95 \%)$ correspondieron a los códigos del CIE-10 que se estimaron como catalogables dentro de causas de MSC. Las patologías certificadas más frecuentes fueron la enfermedad cardíaca isquémica $43 \%$ y las miocardiopatías $27 \%$.

Conclusiones: Este estudio es un primer paso en la caracterización de la MSC en Chile. Los resultados obtenidos demuestran que la enfermedad cardíaca isquémica y las miocardiopatías son las patologías descritas con mayor frecuencia como causa de defunción de MSC en niños y adultos jóvenes, lo que es consistente con la literatura internacional.

Palabras clave: muerte súbita, cardiomiopatía, cardiopatía isquémica. 


\section{Abstract: Causes of sudden death in subjects 1 to 35 years of age in Chile}

There are few events more devastating than Sudden Cardiac Death (SCD) in people without previously known heart disease, especially when occurring during the practice of sports. In Chile we have no reports on SCD epidemiology.

Aim: to describe the incidence of SCD and the underlying pathology in people from 1 to 35 years of age. Methods: We searched the registries from the Department of Statistics and Information of the Ministry of Health (DEIS) and analyzed death certificates from all deaths occurring from 2000 to 2010 in people 1 to 35 years of age. Diagnoses deemed to correspond to SCD were those defined by the ICD-10 codes.

\section{Introducción}

Un paciente que fallece en forma inesperada, sin haber presentado sintomatología previa, ni algún traumatismo que pudiese explicar su deceso, se considera que ha sufrido una muerte súbita no traumática (MS). De las muertes súbitas no traumáticas, alrededor del $90 \%$ son consecuencia de patologías cardiovasculares subyacentes bien definidas $^{1,16}$, grupo considerado como muerte súbita cardíaca (MSC). De acuerdo a la Organización Mundial de la Salud (OMS), la muerte súbita cardíaca es aquella que ocurre antes de 24 horas desde el inicio de la sintomatología. Expertos, sin embargo, la describen como una muerte inesperada, caracterizada por pérdida abrupta de conciencia en un individuo, dentro de la primera hora luego del inicio de la sintomatología o en un paciente sin patología cardíaca previa $^{2}$ o como el paro cardíaco súbito no traumático e inesperado que se produce dentro de 6 horas con un estado de salud previo normal ${ }^{12}$. Es especialmente más frecuente en atletas (la paradoja del deportista), definidos como
Results: Out of 57979 deaths, 1131 (1.95\%) matched the ICD-10 codes estimated as causes of SCD. Ischemic heart disease (43\%) and cardiomyopathies (27\%) were the most frequently certified diagnoses.

Conclusion: This study is a first step in the characterization of SCD in Chile. The results obtained show that ischemic heart disease and cardiomyopathies are the most frequently described causes of SCD in children and young adults, which is consistent with international reports.

Keywords: sudden death, cardiomyopathy, ischemic heart disease un participante de deportes individuales o colectivos de cualquier edad y de programas de entrenamiento de forma regular y sistemática. ${ }^{3,4}$.

En la población general, la MSC tiene una incidencia de $0,001 \%$ a $0,002 \%$ al año (1/100.000-200.000 personas). En atletas, pese a que no es una situación común y que el riesgo de muerte súbita es bajo ${ }^{5,6}$, es 2 a 4 veces más frecuente que en la población general, ocurriendo aproximadamente en 1 en 50.000 a 200.000 atletas anualmente. ${ }^{1,7}$ Se han asociado un número de enfermedades cardiovasculares subyacentes asintomáticas bien definidas, algunas de carácter congénito, como gatillante de un evento de muerte súbita en atletas jóvenes, especialmente en relación al esfuerzo físico desarrollado durante las actividades competitivas. ${ }^{8,9,10}$

Actualmente, en tiempos en que una población progresivamente más sedentaria y obesa es incentivada a realizar actividad física para mejorar su condición cardiovascular, se observa una paradoja consistente en el aumento de la 
mortalidad por muerte súbita cardíaca secundario al aumento de actividad física. ${ }^{11,12}$

En Chile no existen publicaciones que analicen la epidemiología de la MSC, y mucho menos la incidencia en la población deportista joven, por lo que su importancia y real impacto es algo meramente especulativo. Tampoco se conoce de la existencia de algún tipo de registro o catastro nacional de Muerte Súbita, tanto en población general como en subgrupos de interés, tales como atletas o población escolar general.

El objetivo de nuestro estudio fue el de caracterizar la prevalencia de patologías cardíacas y sus subtipos, reconocidos como causantes de MSC en el grupo etario comprendido entre 1 año y 35 años.

\section{Métodos}

Se analizaron los registros de defunciones del Departamento de Estadísticas e Información de Salud (DEIS) del Ministerio de Salud de Chile (MINSAL), los cuales corresponden a bases de datos que incluyen información tanto del Registro Civil e Identificación de Chile y los Certificados de Defunción aportados por el Ministerio de Salud.
Para efectos de nuestro estudio, se realizó un análisis retrospectivo de la información incluida en la base de datos del DEIS, correspondiente a todos los fallecidos en Chile entre los años 2000 y 2010, y que pertenecían al grupo etario entre 1 y 35 años de edad. Se excluyeron los menores de 1 año por ser un subgrupo donde predomina el Síndrome de Muerte Súbita del Lactante entidad sin etiología bien definida y donde no predomina la patología cardiovascular y los mayores de 35 años por ser la enfermedad coronaria la principal causa de muerte súbita de origen cardíaco.

Se seleccionaron los datos de los pacientes fallecidos con diagnósticos relacionados con Muerte Súbita Cardíaca de acuerdo al Código Internacional de Enfermedades (CIE10) clasificadas en Enfermedades del Sistema Circulatorio (Tabla 1). Se incluyeron los códigos R96.0 (muerte instantánea o muerte súbita no explicada en adultos) e I46.1 (muerte cardíaca que excluye enfermedad cardíaca isquémica y trastornos de la conducción). Se consignaron los datos de: edad, sexo y diagnóstico principal de causa de fallecimiento.

Se realizó un análisis retrospectivo descriptivo de los da-

\begin{tabular}{|c|c|c|}
\hline Enfermedad Cardiaca Isquémica & I21.1 a I 21.4 & \\
\hline \multirow[t]{2}{*}{ Miocardiopatía } & I.42 Miocardiopatías & $\begin{array}{l}\text { I42.0 Miocardiopatía dilatada } \\
\text { I42.1 Miocardiopatía hipertrófica obstructiva } \\
\text { I42.2 Miocardiopatía hipertrófica, otras } \\
\text { I42.8 Otras miocardiopatías }\end{array}$ \\
\hline & I40 Miocarditis aguda I.40. 0 Miocarditis infecciosa & I.40.9 Miocarditis aguda inespecífica \\
\hline \multirow[t]{4}{*}{ Trastornos del ritmo } & I.44 Bloqueo Aurículo Ventricular & I.44.2 BAV Bloqueo Aurículo Ventricular completo \\
\hline & I.45 Otros trastornos de la conducción & $\begin{array}{l}\text { I.45.6 Sindrome de Preexcitación } \\
\text { I.45.8 Otros desórdenes conducción inespecificados }\end{array}$ \\
\hline & I.47 Taquicardia paroxística & $\begin{array}{l}\text { I.47.1Taquicardia Paroxística Supraventricular } \\
\text { I.47.2 Taquicardia Ventricular }\end{array}$ \\
\hline & I.49 Otras arritmias cardíacas & I.49.0 Flutter y Fibrilación Ventricular \\
\hline Malformaciones congénitas & $\begin{array}{l}\text { Q.23.0 Estenosis congénita de la válvula aórtica } \\
\text { Q.24.5 Malformaciones de los vasos coronarios }\end{array}$ & \\
\hline \multirow[t]{5}{*}{ Misceláneas } & Q87 & Q.87.4 Síndrome de Marfán \\
\hline & I71 Disección y aneurismas aórticos & $\begin{array}{l}\text { I.71.0 Disección de la aorta (cualquier lugar) } \\
\text { I.71.1 Ruptura aneurisma aorta torácica }\end{array}$ \\
\hline & I27 Otras enfermedades cardiopulmonares & $\begin{array}{l}\text { I.27.0 Hipertensión pulmonar primaria } \\
\text { I.27.2 Otras Hipertensión pulmonar secundaria }\end{array}$ \\
\hline & I46.1 Muerte Súbita cardíaca & \\
\hline & $\begin{array}{l}\text { R96.0 Muerte instantánea } \\
\text { (Muerte súbita inexplicada en adultos) }\end{array}$ & \\
\hline
\end{tabular}




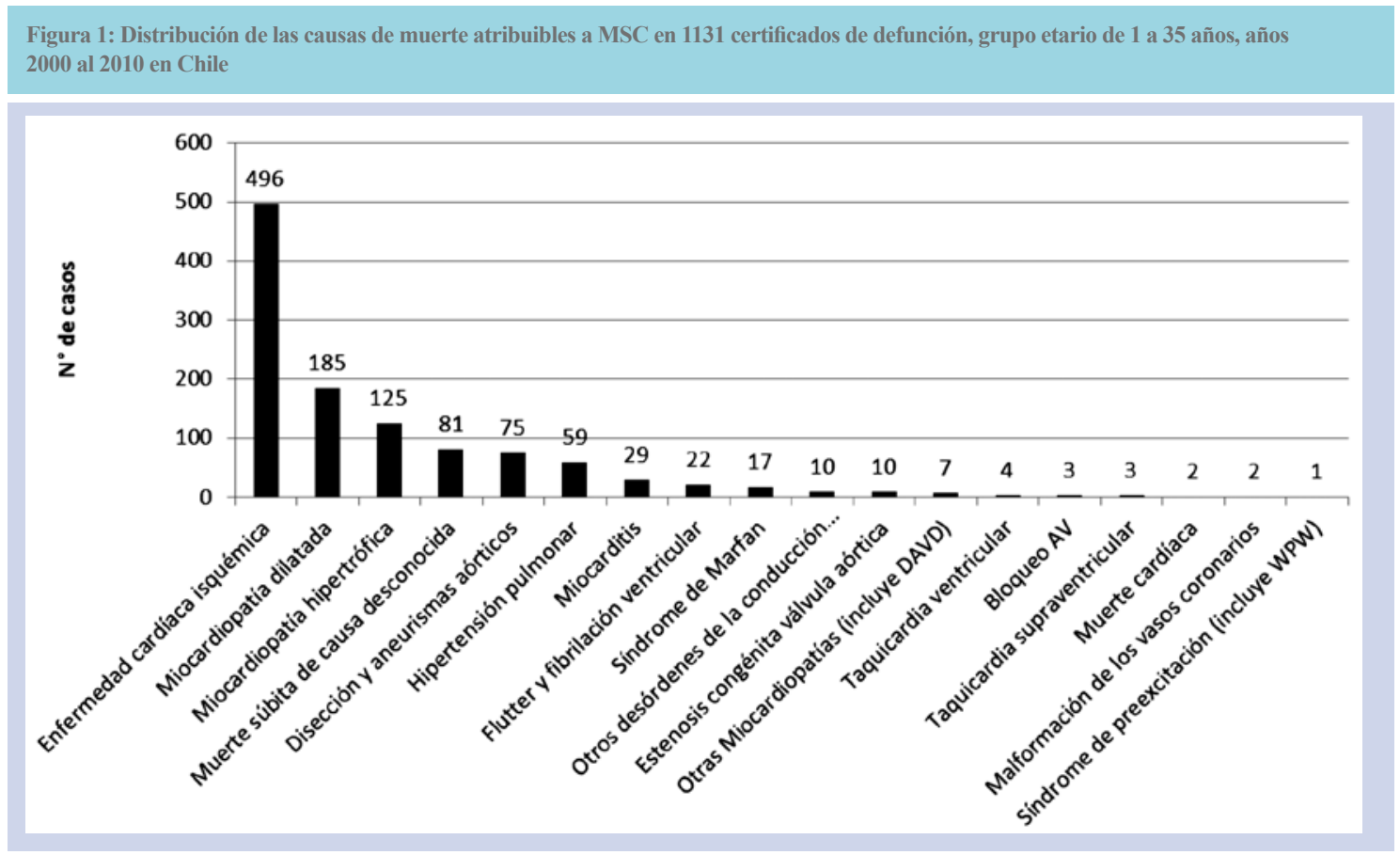

tos, observando qué proporción de las muertes totales corresponden a diagnósticos considerados dentro de MSC; y observando la distribución de los distintos diagnósticos según año de muerte y grupo etario (1 a 35 años).

Los datos fueron extraídos de las bases de datos y tabulados mediante el software Microsoft Excel.

\section{Resultados}

De un total de 956.378 fallecidos en Chile durante el período comprendido entre los años 2000 y 2010, 57.979 $(6,25 \%)$ correspondieron al grupo etario de 1 año a 35 años de edad.

Se observó para el grupo etario analizado una proporción constante entre el total de fallecidos por año y las causas analizadas de MSC, con un promedio de 50 casos anuales para el período analizado.

Del total de fallecidos correspondientes al grupo etario entre 1 año y 35 años, 1.131(1,95\%) presentaron certificados de defunción con diagnósticos de causa de muerte relacionados a los códigos del CIE-10 estimados como catalogables dentro de las causas de MSC buscadas. Las patologías certificadas más frecuentes fueron: enfermedad cardíaca isquémica (I.21) con un $43,8 \%$ y miocardiopatías (I.42) con un $27 \%$ (miocardiopatía dilatada $16 \%$ y miocardiopatía hipertrófica $11 \%$ ), muerte súbita de causa inexplicada (R96.0) (7,16\%), disección y aneurismas aórticos con un 6,6\% y otras (hipertensión pulmonar, miocarditis, fibrilación ventricular, Síndrome de Marfán, entre otras) (Figura 1).

Se observó un aumento evidente en el número de casos en la medida que la edad aumenta y se acerca a los 35 años, ésto dado principalmente por el aumento de los casos de enfermedad cardíaca isquémica (I21) (figura 2).

Existió un claro predominio del género masculino, correspondiendo al $75 \%$ del total de fallecidos $(855$ de 1.131).

En relación al profesional que confeccionó el Certificado de Defunción destaca que en el 56\% de los casos fue realizado por médico cuyo código indica "legista o patólogo", pero no se especifica en qué casos estos médicos realizaron autopsia.

\section{Discusión}

Del total de fallecidos en Chile (956.378) durante el período comprendido entre los años 2000 y 2010, 1131(1,95\%) presentaron certificados de defunción con diagnósticos de causa de muerte relacionados a los códigos del CIE-10 estimados como catalogables dentro de las causas de MSC. La distribución del número de casos anuales fue semejante en todos los años estudiados, con tendencia a mantener- 


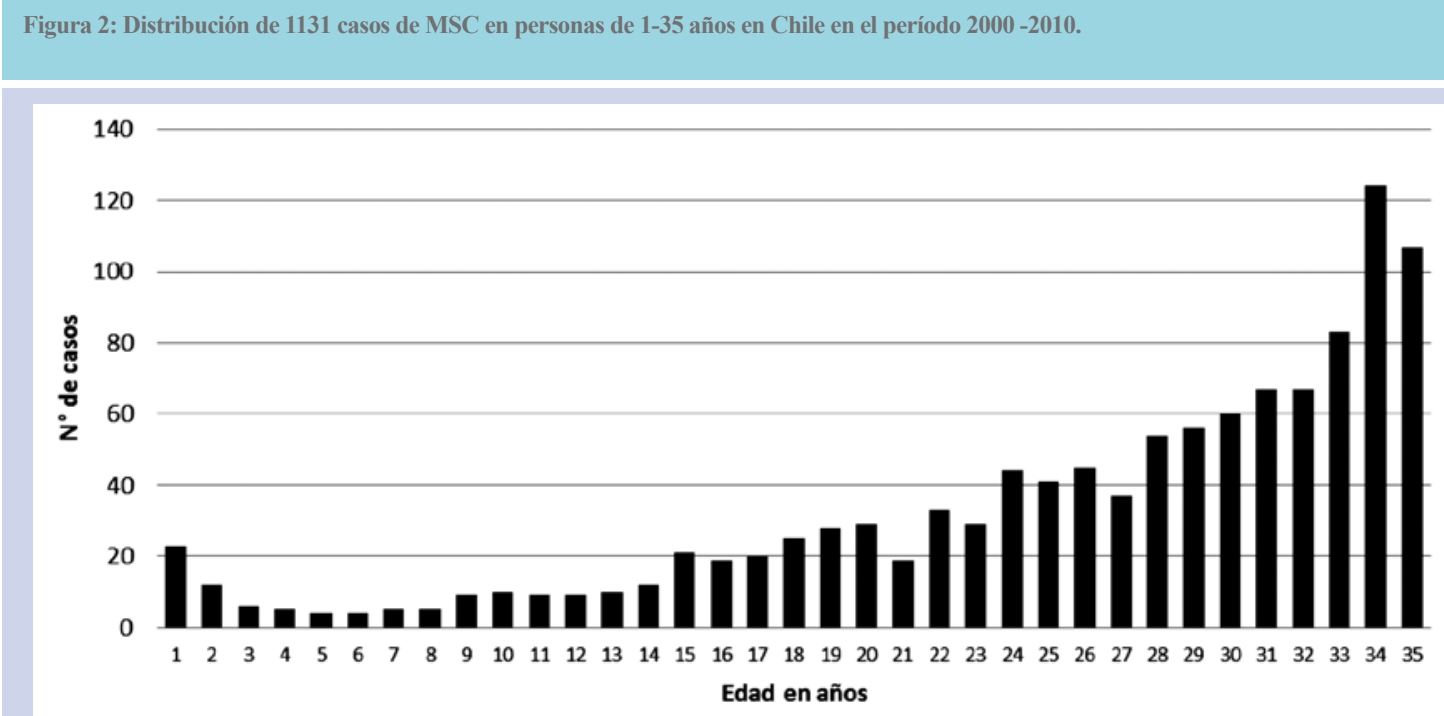

se estable en el tiempo tanto el número total de fallecidos entre 1-35 años como el número que corresponde a los códigos CIE-10 analizados, variando entre 41 y 60 casos anuales, con un promedio de 50 casos al año para el período analizado.

Los datos obtenidos presentan una distribución semejante a estudios internacionales ${ }^{13,14,15}$ en cuanto al aumento del número de casos al ir aumentando la edad, la diferencia según género con claro predominio de los casos en hombres y la mayor prevalencia de enfermedad cardíaca isquémica y las miocardiopatías por sobre las otras causas. Debe destacarse que la incidencia de enfermedad cardíaca isquémica en estos grupos etarios como primera causa de MSC hace relevante su evaluación en detalle dado que pudieran ser factores de riesgo cardiovascular que están manifestándose en forma más precoz y/o pudiera estar asociado con el consumo de sustancias ilícitas (cocaína o sus derivados) en su génesis.

La gran mayoría de las condiciones cardiovasculares detectadas en este estudio como causantes de MSC y que se vinculan con la actividad deportiva pueden ser detectadas precozmente por medio del control de factores de riesgo (cardiopatía isquémica) o evitando conductas de riesgo (consumo de drogas ilícitas) y por medio de la realización de evaluaciones preparticipativas, dirigidas a la búsqueda de aquellas condiciones cardiovasculares subyacentes que se pueden manifestar durante la práctica deportiva.
Limitaciones del estudio: en primer lugar, sólo se tuvo acceso a bases de datos ya procesadas de certificados de defunción, siendo el ideal que los revisores tengan acceso directo a los mismos certificados para clasificar cuales corresponden con mayor certeza a los criterios buscados para catalogar como MSC $\mathrm{MC}^{13,14}$. En segundo lugar, el certificado de defunción presenta importantes limitaciones ya que no sabemos detalles de los fallecimientos tales como relación con ejercicio, consumo de drogas, etc., en especial en aquellos casos en que el fallecimiento fue de causa indeterminada. Existen países tales como Dinamarca donde es obligatorio colocar con texto libre detalles relevantes que pueden permitir aclarar las circunstancias que rodearon la muerte, teniendo esto implicancias tanto médicas como legales.

\section{Conclusiones}

Este es un primer estudio en la búsqueda de la caracterización de la MSC en Chile. Observamos que existe en forma regular un promedio de 50 casos anuales al año de muertes cuyas causas pueden ser correlacionadas con MSC en nuestro país.

Los resultados obtenidos demuestran que la enfermedad cardíaca isquémica y las miocardiopatías son las patologías descritas con mayor frecuencia, siendo esto consistente con la literatura internacional. Esto enfatiza la importancia del desarrollo de programas de Prevención 
cardiovascular y de Evaluación pre participativa en actividades deportivas.

Otro punto a desarrollar es el énfasis en llenar en forma adecuada y completa los certificados de defunción, ya que son fuente de información fundamental en la toma de decisiones desde el punto de vista de políticas de Salud
Pública.

Es fundamental realizar un trabajo colaborativo que permita confeccionar un catastro nacional de las MSC, con definiciones claras y evaluaciones clínicas y anatomopatológicas lo más completas posibles para así poder desarrollar estrategias preventivas en el futuro.

\section{Referencias:}

1. FERREIRA M, SANTOS-SILVA PR, DE ABREU LC, VALENTI VE, CRISPIM V, IMAIZUMI C, et al. Sudden cardiac death athletes: a systematic review. Sports Med Arthrosc Rehabil Ther Technol 2010; 2: 19

2. AMSTERDAM EA . Sudden death during exercise.Cardiology 1990;77: 411-417.

3. ALBERT CM, CHAE CU, GRODSTEIN F, ROSE LM, REXRODE KM, RUSKIN JN, et al. Prospective study of sudden cardiac death among women in the United States. Circulation $2003 ; 107$ : 2096-2101.

4. OWOEYE, OB. Pattern and management of sports injuries presented by Lagos state athletes at the 16th National Sports Festival (KADA games 2009) in Nigeria. Sports Med Arthrosc Rehabil Ther Technol 2010;2:3.

5. VAN CAMP SP, BLOOR CM, MUELLER FO, CANTU RC, OLSON HG . Nontraumatic sports death in high school and college athletes. Med Sci Sports Exerc 1995; 25:641-647.

6. MARON BJ, GOHMAN TE, AEPPLI D. Prevalence of sudden cardiac death during competitive sports activities in Minnesota high school athletes. J Am Coll Cardiol 1998; 32:1881-1884

7. MARON BJ, SHIRANI J, POLIAC LC, MATHENGE R, ROBERTS WC, MUELLER FO. Sudden death in young competitive athletes: clinical, demographic, and pathological profiles. JAMA 1996; 276 : 199-204.

8. MARON BJ. Cardiovascular risks to young persons on the athletic field. Ann Intern Med 1998; 129: 379-386.

9. THIENE G, PENNELLI N, ROSSI L. Cardiac conduction system abnormalities as a possible cause of sudden death in young athletes. Hum Pathol 1983;14:706-709.

10. BASSO C, MARON BJ, CORRADO D, THIENE G. Clinical profile of congenital coronary artery anomalies with origin from the wrong aortic sinus leading to sudden death in young competitive athletes. J Am Coll Cardiol 2000;35:1493-501.

11. MARON BJ. Sudden Death in Young Athletes. N Engl J Med 2003;349: 1064-1075

12. MARON BJ, EPSTEIN SE, ROBERTS WC. Causes of sudden death in competitive athletes. J Am Coll Cardiol 1986;7:204-14

13. WINKEL BG, HOLST AG, THEILADE J, KRISTENSEN IB, THOMSEN JL, OTTESEN GL, et al. Nationwide study of sudden cardiac death in persons aged 1-35 years. Eur Heart J 2011; 32: 983-990

14 MARGEY R, ROY A, TOBIN S, O'KEANE CJ, MCGORRIAN C, MORRIS V, et al. Sudden cardiac death in 14to 35-year olds in Ireland from 2005 to 2007: a retrospective registry. Europace 2011;13: 1411-1418

15. PAPADAKIS M, SHARMA $S$, COX S, SHEPPARD MN, PANOULAS VF, BEHR ER. The magnitude of sudden cardiac death in the young: a death certificate-based review in England and Wales. Europace 2009; 11: 1353-1358

16. DÍAZ F, MERCADO C, TRONCOSO I, HEUSSER F, CLAVERÍA C. Rev Med Chile 2010; 138: 223-232 